\title{
A Medley with over ten years of (mostly) light-ion production measurements at The Svedberg Laboratory
}

\author{
S. Pomp ${ }^{1, \text { a }}$, R. Bevilacqua ${ }^{1}$, M. Hayashi ${ }^{2}$, S. Hirayama ${ }^{2}$, F.-R. Lecolley ${ }^{3}$, N. Marie ${ }^{3}$, \\ Y. Naitou ${ }^{2}$, U. Tippawan ${ }^{4}$, Y. Watanabe ${ }^{2}$, P. Andersson ${ }^{1}$, J. Blomgren ${ }^{1, b}$, C. Gustavsson ${ }^{1}$, \\ A. Hjalmarsson ${ }^{5}$, A.V. Prokofiev ${ }^{5}$, V.D. Simutkin ${ }^{1}$, E. Tengborn ${ }^{1}$, M. Tesinsky ${ }^{6}$, M. Österlund ${ }^{1}$, \\ and A. Kolozhvari ${ }^{7}$ \\ 1 Department of Physics and Astronomy, Uppsala University, Box 516, 75120 Uppsala, Sweden \\ 2 Department of Advanced Energy Engineering Science, Kyushu University, Fukuoka, Japan \\ 3 LPC, ENSICAEN, Université de Caen, 14050 Caen Cedex, France \\ 4 Plasma and Beam Physics Research Facility, Chiang Mai University, Chiang Mai, Thailand \\ 5 The Svedberg Laboratory, Uppsala University, Box 533, 75121 Uppsala, Sweden \\ 6 Department of Nuclear and Reactor Physics, Royal Institute of Technology, Stockholm, Sweden \\ 7 Saint Petersburg State University, Saint Petersburg, Russia
}

\begin{abstract}
Over the past years an experimental programme has been run at the neutron beam of The Svedberg Laboratory with the aim to study light-ion production induced by 96 and $175 \mathrm{MeV}$ neutrons for a wide variety of targets. The measurements have been conducted using the Medley facility which allows measurement of p, d, t, He-3 and alpha production at fixed angles (from 20 to 160 degrees in steps of 20 degrees) over a wide dynamic range. An overview of the results obtained at the now finished campaign at $96 \mathrm{MeV}$ will be given. Since 2007 we have been running at $175 \mathrm{MeV}$ with $\mathrm{C}, \mathrm{O}, \mathrm{Si}, \mathrm{Fe}, \mathrm{Bi}$ and $\mathrm{U}$ as target material. Preliminary results from these measurements will be shown and compared to model calculations with Talys-1.2. We also summarize the Medley measurements of elastic $n p$ and $n d$ scattering and of angular distributions of fission fragments.
\end{abstract}

\section{Introduction}

The Medley setup was initially designed for neutron-induced light-ion production measurements at energies up to $100 \mathrm{MeV}$. The purpose was to measure needed nuclear data for medical applications such as fast neutron cancer therapy at the neutron beam of The Svedberg Laboratory (TSL). The detector arrangement consists of eight three-element telescopes mounted inside an evacuated scattering chamber. The telescopes are placed to measure at angles between 20 and 160 degrees, in steps of 20 degrees. Particle identification is achieved by means of the $\Delta \mathrm{E}-\Delta \mathrm{E}-\mathrm{E}$ method, using two silicon surface barrier detectors (SSBD) followed by a CsI(Tl) scintillator. The thickness of the first SSBD is about 50 to $60 \mu \mathrm{m}$. Thus, it is possible to measure double-differential cross sections of the produced light ions over a wide dynamical range. Details about the set-up are given in Ref. [1] and a photograph is shown in Fig. 1.

Soon after the first measurements on carbon have been done, other applications came into focus. These comprise mainly accelerator-based transmutation of nuclear waste and soft-error effects in computer memory [2]. In addition to the light-ion production studies, measurements of elastic $n p$ and $n d$

\footnotetext{
a e-mail: stephan.pomp@physics.uu.se

b Now at Vattenfall AB.
}

This is an Open Access article distributed under the terms of the Creative Commons Attribution-Noncommercial License 3.0, which permits unrestricted use, distribution, and reproduction in any noncommercial medium, provided the original work is properly cited. 

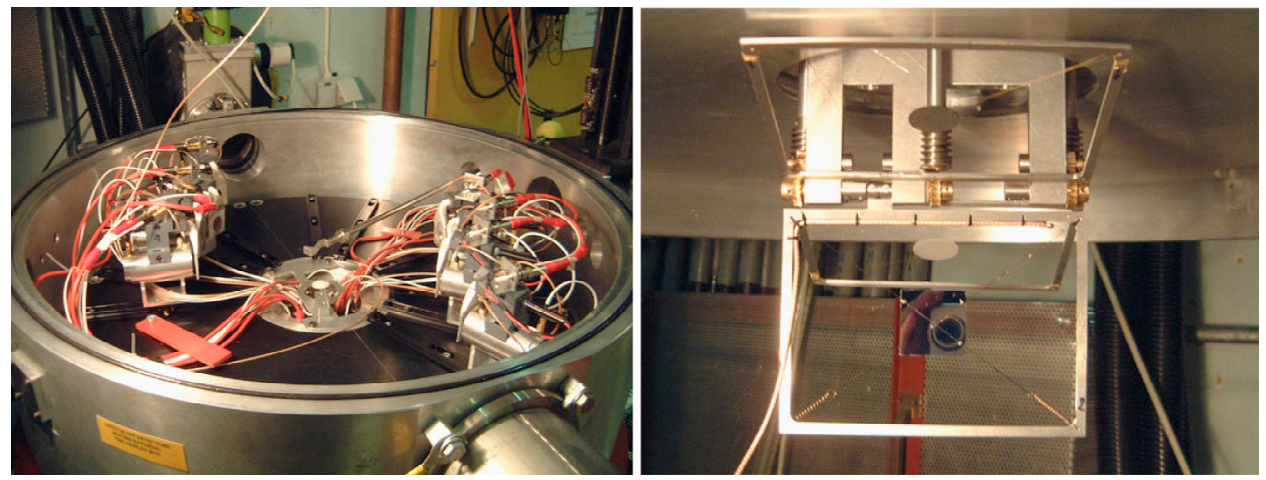

Fig. 1. Left picture: Photograph of the opened Medley chamber with inserted Am-241 calibration source at the target position. Neutrons enter from the opening seen in the upper half of the picture. The exit flange is seen on the lower right side. Right picture: Photograph of the targets mounted inside aluminium frames on the lock of the chamber. During an experimental run, the targets can be changed from the outside without breaking the vacuum.

scattering as well as angular distributions of fission yields have been done. In Sect. 2 we summarize the light-ion campaign at $96 \mathrm{MeV}$ and Sect. 3 describes the ongoing campaign at $175 \mathrm{MeV}$ with an upgrade version of the original set-up. Sect. 4 summarizes the elastic scattering and fission measurements. We conclude with a summary and an outlook on possible future activities.

\section{Light-ion production measurements at $96 \mathrm{MeV}$}

The $96 \mathrm{MeV}$ measurements have been performed at the old quasi-monoenergetic neutron beam of TSL [1]. Neutrons were produced by the ${ }^{7} \mathrm{Li}(\mathrm{p}, \mathrm{n})$ reaction. The experimental set-up was located about $9 \mathrm{~m}$ downstream the Li target and the time-of-flight (TOF) method was used to suppress the neutron tail. The arrangement of collimators and a clearing magnet allowed for an almost background free measurement at sufficient beam intensity. Measurements have been done on C [3], O [4], Si [2], Fe, $\mathrm{Pb}$ and $\mathrm{U}[5]$.

The main corrections that are necessary to apply are (1) correction for the reaction losses in the CsI detector, (2) correction of energy and particle loss inside the target, and (3) correction for the so-called frame overlap or wrap around. The correction for the reaction losses has been determined from MCNP and Geant simulations and amounts to at most $8 \%$. A detailed description of the target correction is given in Ref. [6]. For the frame overlap correction we have determined the (wrongly) accepted neutrons of lower energy from measuring elastic $n p$ scattering with a $\mathrm{CH}_{2}$ target (see Fig. 2). Corrections have then been done using tabulated values from the ICRU Report 63 [7]. Even after correction for the frame overlap, the accepted neutron spectrum is not truly monoenergetic but has a small remaining tail contribution [4].

From the double differential cross sections for the various targets, we also deduced the energy and angular differential cross sections, particle production cross sections and Kerma coefficients. The latter showed that the evaluation in Ref. [7] underestimates the $\mathrm{C}$ and $\mathrm{O}$ kerma at $96 \mathrm{MeV}$ by about $10 \%$ [8].

\section{Light-ion production measurements at $175 \mathrm{MeV}$}

In 2004 the new neutron beam at TSL was taken into operation. A reduced distance from the Li target now allows measurements at the highest available energy of TSL within reasonable data-acquisition times [9]. The detectors within Medley had to be adapted to the increased energy range; now $1000 \mu \mathrm{m}$ thick SSBD are used as $\Delta \mathrm{E}_{2}$ detector and longer $\mathrm{CsI}(\mathrm{Tl})$ have been installed (see, e.g., Ref. [10]). A measurement of the $\mathrm{CsI}(\mathrm{Tl})$ response to $160 \mathrm{MeV}$ protons has been done at the proton beam line of TSL. The experimental results are in good agreement with model calculations [11]. 


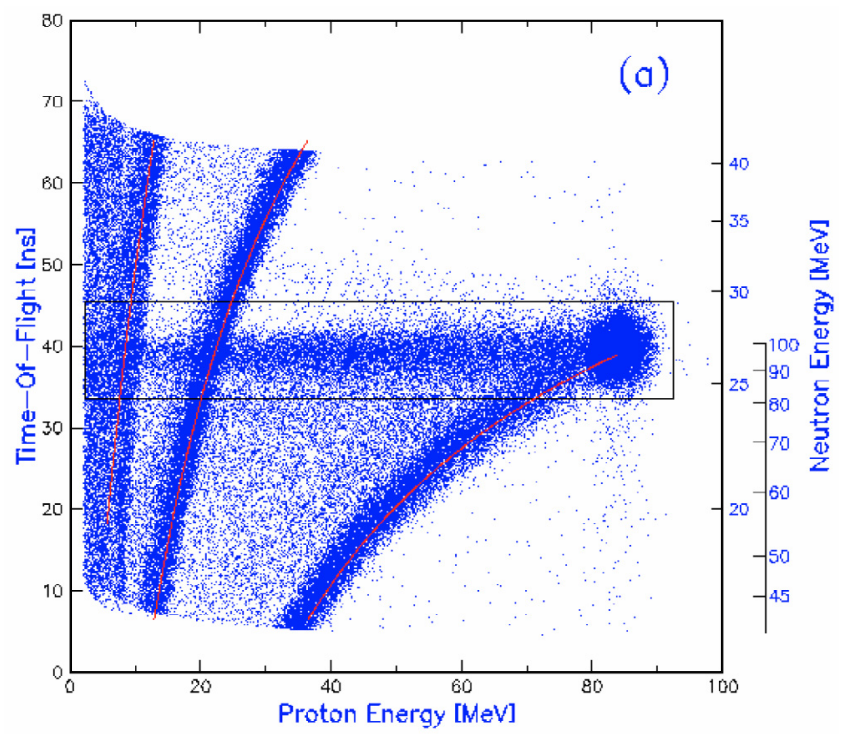

Fig. 2. Measured proton energy versus relative neutron TOF for elastic $n p$ scattering from a $\mathrm{CH}_{2}$ target. The horizontal band stems from ${ }^{12} \mathrm{C}(\mathrm{n}, \mathrm{px})$ events. The bent band reflects the kinematic relation between neutron and proton energy for a fixed scattering angle. Frame overlap can be seen at proton energies of about 8 and $20 \mathrm{MeV}$.

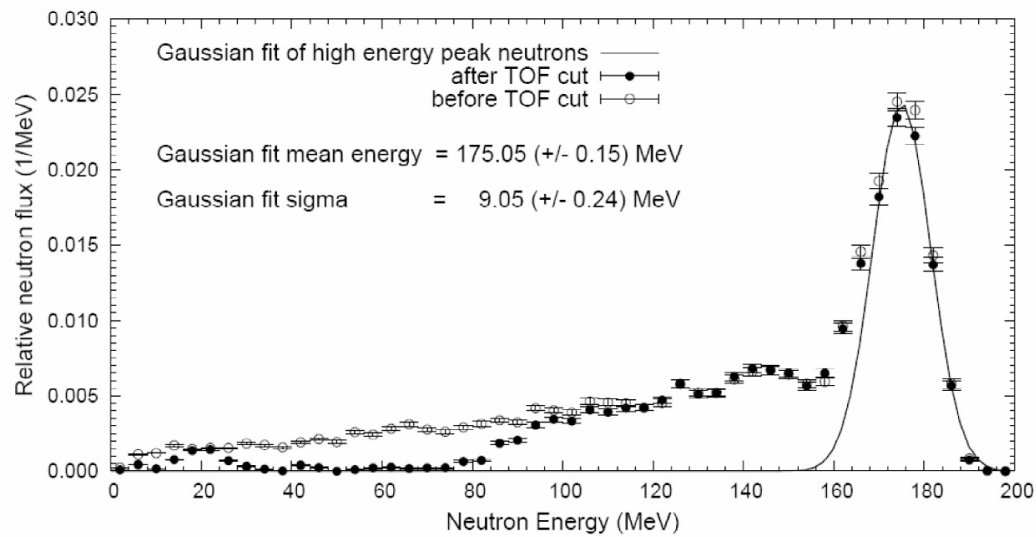

Fig. 3. Example of a quasi-monoenergetic $175 \mathrm{MeV}$ neutron spectrum as measured with Medley (open circles). Closed circles show the accepted neutron spectrum after application of the TOF cut. It can be seen that the TOF cut is effective only below $90 \mathrm{MeV}$. The small peak at $20 \mathrm{MeV}$ is from frame overlap.

Due to the limited time resolution of the SSBD, the width in time of the proton beam pulse onto the ${ }^{7} \mathrm{Li}$ target, and, most of all, the much reduced distance between Li target and the target in Medley, the effect off the TOF cut on the accepted neutron spectrum is, compared to the runs at $96 \mathrm{MeV}$, much reduced. An example for a neutron spectrum before and after the TOF cut is shown in Fig. 3.

So far data have been collected for the following targets: C, O, Si, Fe, Bi and U. Preliminary results have, e.g., been published in Refs. [10-13]. Some preliminary results for p, d, t, He-3 and alpha particle production from $\mathrm{Fe}$ and $\mathrm{Bi}$ at 20 degrees are also shown in Figs. 4 and 5 and compared to the corresponding measurement at $96 \mathrm{MeV}$.

These preliminary data indicate that the Talys code [14] manages to describe proton production reasonably well even at $175 \mathrm{MeV}$ but has problems with the composite particles. Two problems that seem to remain even for protons are the description of the compound part of the spectrum and the 


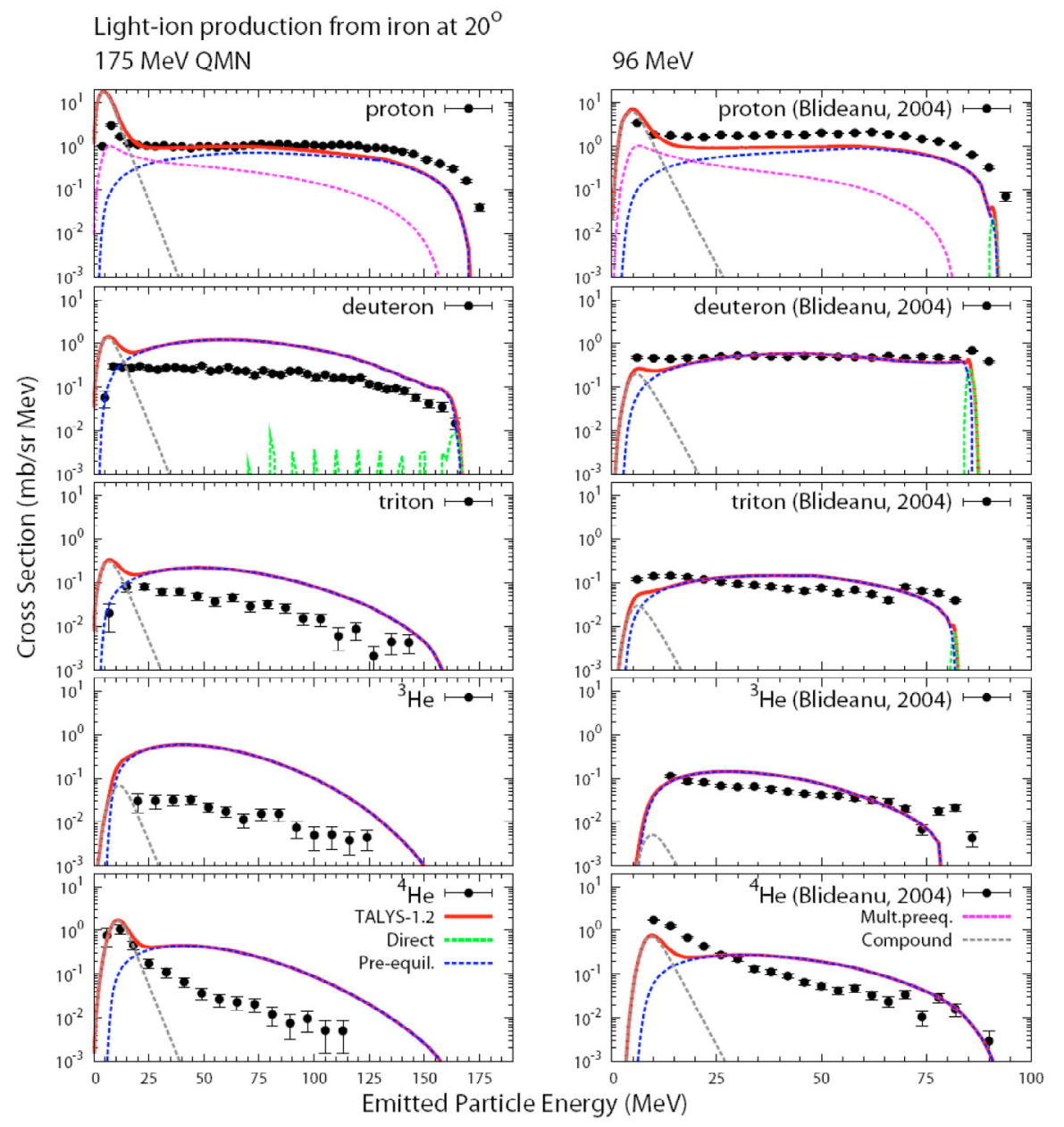

Fig. 4. Preliminary results for light-ion production (from protons, top row to alpha particles, bottom row) cross sections at 20 degree from iron at $175 \mathrm{MeV}$ (filled circles, left side) compared to the results from Ref. [5] at $96 \mathrm{MeV}$ (filled circles, right side). The solid and dashed lines indicate the Talys-1.2 calculations [14] with the relative contributions of different reaction mechanisms.

behaviour at the high-energy end of the spectrum (especially for $\mathrm{Fe}(\mathrm{n}, \mathrm{px})$ ). The latter can only partially be explained by limited experimental energy resolution.

\section{Elastic $n p$ and $n d$ scattering and fission measurements}

While light-ion production has been the main research line for which Medley has been used, but also other measurements have been done. Elastic $n p$ scattering has always been necessary for normalization and spectrum measurement purposes. It has also been used for spectrum measurements of the newly installed white neutron source ANITA from the maximum energy of close to $180 \mathrm{MeV}$ down to about $1.5 \mathrm{MeV}$ [12]. However, even dedicated runs to measure both the elastic $n p$ and $n d$ scattering cross sections have been performed. For the latter a $\mathrm{CD}_{2}$ plastic target has been used. This target was mounted to be almost parallel with the beam axis to minimize energy loss for the outgoing deuterons at high angle. In this way the cross sections could be obtained over a wide angular range. The results showed clear effects from three-body force effects [15-17].

Also efforts towards measuring angular distributions of fission fragments have been undertaken. For these experiments we have used ${ }^{238} \mathrm{U}$ evaporated on a $\mathrm{CH}_{2}$ backing as target. The fission fragments 


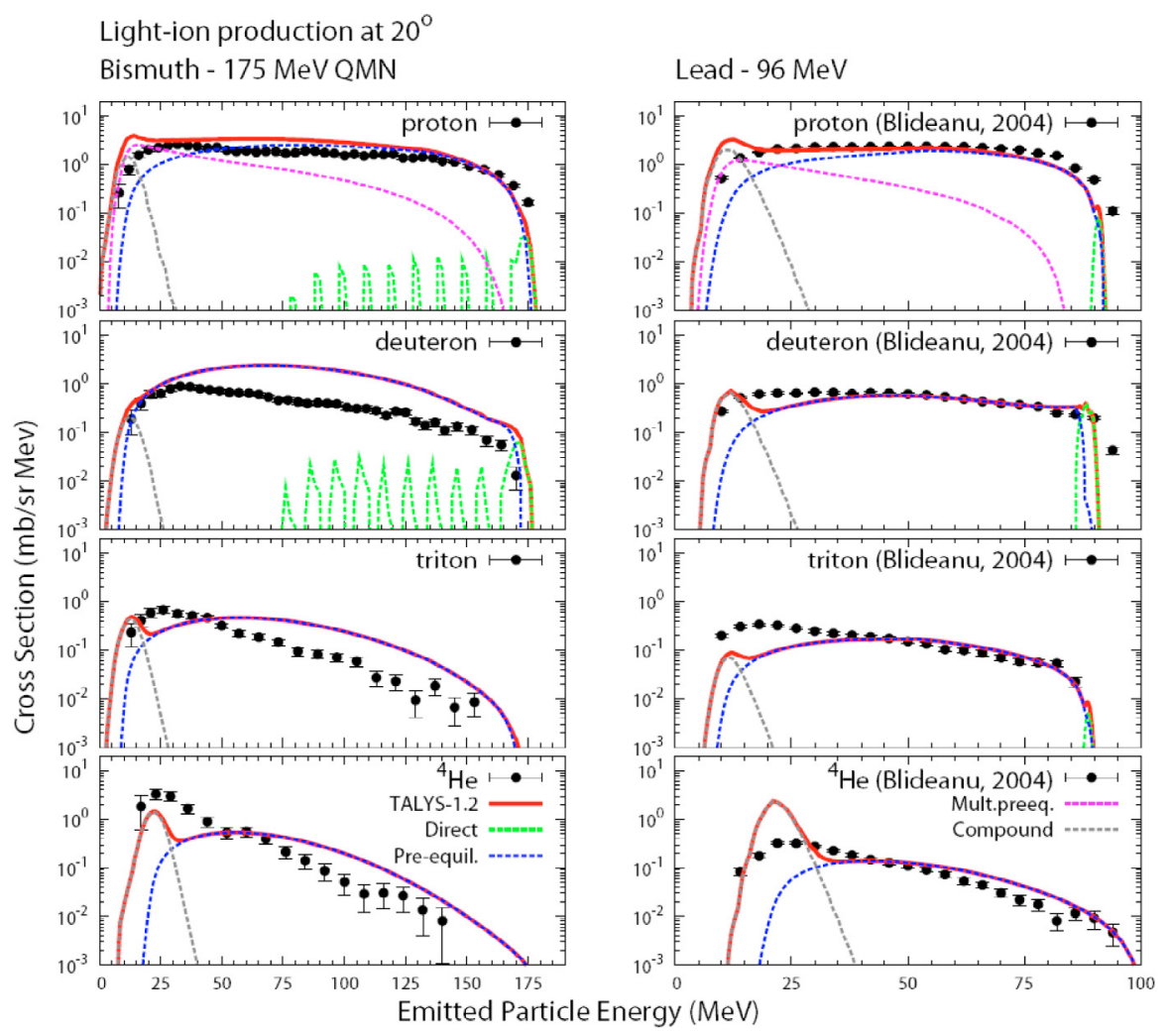

Fig. 5. Preliminary results for light-ion production (from protons, top row to alpha particles, bottom row) cross sections at 20 degree from bismuth at $175 \mathrm{MeV}$ (filled circles, left side) compared to the results from Ref. [5] at $96 \mathrm{MeV}$ for a lead target (filled circles, right side). The solid and dashed lines indicate the Talys-1.2 calculations $[\mathrm{xx}]$ with the relative contributions of different reaction mechanisms.

were registered in the first SSBD and the 20 degree telescope registered, in addition, elastic $n p$ scattering events from the backing. During beam-off alpha particles from alpha decay have been counted with the same detector arrangement. With this method, normalisation problems like amount of target mass and effective solid angle was avoided and the anisotropy for neutron-induced fission of ${ }^{238} \mathrm{U}$ at $21 \mathrm{MeV}$ could be measured [18]. The achievable time resolution with Medley at the TSL neutron beam was a limiting factor for further progress.

\section{Summary and outlook}

We have briefly summarized the experimental efforts for which the Medley set-up has been used at the TSL neutron beams. A large data set on neutron-induced light-ion production at 96 and $175 \mathrm{MeV}$ was obtained for the first time. Thus, together with the results from elastic scattering measurements with the SCANDAL set-up (not discussed here but see, e.g., Ref. [19]), nuclear model codes dealing with the tricky challenge to link the low energy region $\left(E_{n}<20 \mathrm{MeV}\right)$, where experimental data are readily available, with the high energy domain $\left(E_{n}>200 \mathrm{MeV}\right)$, where different reaction mechanisms are expected to dominate, can now be compared with extensive high-quality experimental data.

For the future we plan to closer explore the neutron energy domain below $30 \mathrm{MeV}$. This is, again, driven by lack of experimental data in combination with both nuclear data needs for applications such as gas production in construction material in nuclear energy production (both so-called GenIV and fusion reactors), soft-error effects in microelectronics, etc., and persisting problems of nuclear model codes to describe, e.g., pre-equilibrium emission and handling of composite particles. Therefore, we 


\section{EPJ Web of Conferences}

plan a new campaign with an adapted version of Medley at the future Neutrons For Science (NFS) facility at Spiral2/GANIL, France. There, we also plan to renew our efforts on fission measurements with improved timing capabilities of the future Medley.

Many people have contributed to the work summarized here and financial support has been received from many different organizations. Here we would like to mention especially the support received for the latest measurements at $175 \mathrm{MeV}$ :

This work has been supported by the Swedish Radiation Safety Authority, the Swedish Nuclear Fuel and Waste Management Company, Ringhals AB within the NEXT project, by the European Commission within the Sixth Framework Programme through "European Facilities for Nuclear Data Measurements" (EFNUDAT), contract number FP6-036434, and by a Grant-in-Aid for human resource development from Kyushu Industrial Technology Center and Sasakawa Scientific Research Grant from the Japan Science Society.

Special thanks are, as always, due to the staff of TSL for their excellent support before, during and after the experiment.

\section{References}

1. S. Dangtip, et al., Nucl. Instr. Meth. A 452, 484 (2000)

2. U. Tippawan, et al., Phys. Rev. C 69, 064609 (2004)

3. U. Tippawan, et al., Phys. Rev. C 79, 064611 (2009)

4. U. Tippawan, et al., Phys. Rev. C 73, 034611 (2006)

5. V. Blideanu, et al., Phys. Rev. C 70, 014607 (2004)

6. S. Pomp, U. Tippawan, Nucl. Instr. Meth. A 572, 893 (2007)

7. ICRU Report 63, Nuclear data for neutron and proton radiotherapy and for radiation protection. (Bethesda, MD: ICRU, 2000)

8. M. Göttsche, et al., $11^{\text {th }}$ Neutron and Ion Dosimetry Symposium (NEUDOS-11), Cape Town, October 12-16, 2009, South Africa, accepted for publication in Rad. Meas.

9. A.V. Prokofiev, et al., Radiat. Prot. Dosim. 126, 18-22 (2007)

10. R. Bevilacqua, et al., Proceedings of the International Conference on Nuclear Data for Science and Technology (ND 2010), Jeju Island, South Korea (to appear)

11. M. Hayashi, et al., Engineering Science Reports, Kyushu Univ. 29 [4], 374-378 (2008) (in Japanese)

12. Y. Naitou, et al., Proceedings of the 2009 Annual Symposium on Nuclear Data, Ricotti, Tokai, Japan, Nov. 26-27, 2009

13. S. Hirayama, et al., Proc. of the Fifth Int. Symp. on Radiation Safety and Detection Technology (ISORD-5), Kitakyushu, Japan, July 15-17, 2009

14. A.J. Koning, S. Hilaire, M.C. Duijvestijn, Proceedings of the International Conference on Nuclear Data for Science and Technology (ND 2007), Nice, France, pp. 211-214 (EDP Sciences, 2008)

15. P. Mermod, et al., Phys. Lett. B 597, 243 (2004)

16. P. Mermod, et al., Phys. Rev. C 72, 061002(R) (2005)

17. P. Mermod, et al., Phys. Rev. C 74, 054002 (2006)

18. A. Prokofiev, et al., Proceedings of the International Conference on Nuclear Data for Science and Technology (ND2004), Santa Fé, AIP Conference Proceedings No. 769, pp. 800-803 (Melville, New York, 2005)

19. A. Öhrn, et al., Phys. Rev. C 77, 024605 (2008) 